assess the prevalence and predictors of major depressive disorder (MDD) among women living with HIV in rural settings in India. Methods A cross-sectional sample of 905 HIV-infected rural women aged between 18 and 49 years was recruited from District Anti Retroviral Therapy centre. Trained psychiatric social workers interviewed participants for perceived stigma, stress, social support, quality of life and also screened for depression. Those who had a score of 6 or above on Self Rating Questionnaire (SRQ) were further evaluated by for Major Depressive Disorder (MDD) using Mini International Neuropsychiatric Interview (MINI.5.0.0) by mental health professionals experienced in administering psychiatric rating scales.

Results The sample consisted of participants with mean age of $36.69 \pm 7.06$ years, with largely uneducated $(17.9 \%)$ or studied less than $7^{\text {th }}$ grade $(65.7 \%)$ and unskilled labourers $(81.9 \%)$. About half of the respondents had lost their spouses due to HIV infection (45.5\%). Majority of the respondents disclosed their HIV status only to their closest relatives $(82.2 \%)$. In addition, they perceived it is risk to disclose their status to others (86.5\%).

The prevalence of MDD was $19.6 \%$. The MDD group had significantly higher scores for perceived stigma $(p=0.008)$, stress $(\mathrm{p}=0.08)$ and a lower score for social support $(\mathrm{p}<$ 0.0001) compared to the non-depressed group. Further, on regression analysis, higher scores for stigma $(\mathrm{p}=0.004)$, stress $(p<0.0001)$ and lower scores for social support $(p=0.004)$ predicted MDD.

Conclusions MDD is associated with various modifiable psychosocial risk factors among women with HIV. The study highlights that there is a need for developing a context appropriate psycho-social intervention to target depression among women living with HIV.

\subsection{OVERLAPPING HIV AND SEX WORK STIGMA: EXPERIENCES FROM 14 SITES ACROSS ZIMBABWE}

${ }^{1} \mathrm{JR}$ Hargreaves*, ${ }^{2} \mathrm{~J}$ Busza, ${ }^{3} \mathrm{P}$ Mushati, ${ }^{1} \mathrm{E}$ Fearon, ${ }^{3,4} \mathrm{FM}$ Cowan. ${ }^{1}$ Department of Social and Environmental Health Research, London School of Hygiene and Tropical Medicine, London, UK; ${ }^{2}$ Department of Population Health, London School of Hygiene and Tropical Medicine, London, UK; ${ }^{3}$ Centre for Sexual Health and HIVIAIDS Research Zimbabwe, Harare, Zimbabwe; ${ }^{4}$ Department of Infection and Population Health, University College London, London, UK

\subsection{6/sextrans-2015-052270.123}

Background Stigma remains a barrier to female sex workers' (FSW) access to health services, reflecting fears of being identified as engaging in a criminalised and marginalised occupation, and discrimination and mistreatment by health workers. For FSW living with HIV, the additional stigma can exacerbate discrimination and reluctance to seek care.

Methods We describe intersecting patterns of anticipated and experienced stigma related to sex work and HIV status among FSW in Zimbabwe. As part of the baseline survey for the SAPPH-IRe cluster-randomised trial, we recruited 2722 FSW in 14 sites using Respondent Driven Sampling. We asked 9 questions on perceived sex-work-related stigma. Women self-identifying as HIV+ $(\mathrm{n}=1011)$ answered an additional stigma scale.

Results Sex work-related stigma was higher than HIV-related stigma. This held true for internalised, perceived and experienced forms. For instance, 37\% of FSW reported "feeling ashamed" due to their occupation, compared to $20 \%$ of those with HIV feeling shame due to their status; 59\% of FSW felt they had "lost respect or standing" as a result of being sex workers, while 23\% among the HIV+ felt HIV had reduced their social status. In relation to being "talked about badly" for being FSW or HIV+, the figures were $47 \%$ and $12 \%$. Similarly, $19 \%$ of respondents reported being insulted as FSW but just $5 \%$ of those living with HIV felt insulted due to their status. Denial of services by health care workers was low, but nonetheless considered higher for being a sex worker compared to being HIV+. Conclusions Sex workers in Zimbabwe have high HIV prevalence and experience layered stigma for their role as "immoral" women as well as "vectors of disease." That sex-work related stigma is more pervasive than HIV-related stigma may be due to "normalisation" of HIV following introduction of widespread treatment, with comparatively greater disapproval for sex work.

Disclosure of interest statement The SAPPH-IRe trial is using Truvada donated by Gilead. We have no other relationships with commercial entities to disclose.

\section{O08.4 THE ISEAN HIVOS STIGMA AND DISCRIMINATION STUDY (SADS) IN HEALTH CARE SETTINGS (SADS-HCS- 2015) IN SOUTH EAST ASIA ISLAND COUNTRIES - INITIAL FINDINGS FROM INDONESIA}

${ }^{1}$ Loyd Brendan P Norella*, ${ }^{2}$ Maria Cristina V Ignacio. ${ }^{1}$ Program Director, ISEAN Hivos Program; ${ }^{2}$ Independent Consultant- ISEAN Hivos Program

\subsection{6/sextrans-2015-052270.124}

A four-country study was conducted to provide information on the status of stigma and discrimination (SAD) among Men who have Sex with Men (MSM) and Transgender (TG) persons in Indonesia, Malaysia, the Philippines and Timor Leste. Based on the Indicators described in the Stigma and Discrimination Index Questionnaire, this study focused on their experiences with local health care workers and in various health care settings such as clinics, health centres and hospitals. A total of 2,412 respondents participated with 1,000 representatives from Indonesia. This abstract focuses on the initial findings of the SADS-HCS-2015 in Indonesia.

Field surveys were conducted by trained members of 18 community based organisations from across Indonesia. A 12-question paper-based or electronic questionnaires were employed to reach out to MSM and TG clients of health clinics and hospitals (both private and public) using convenience sampling. Stigma and discrimination were described in terms of the respondents' selfreported perception of: 1 . Refusal of health care services, 2. Physical maltreatment, 3. Verbal maltreatment and 4. Provision of health care service below standards. Results indicate that Verbal Maltreatment was the most common experience $(n=50)$ followed by Provision of health service below standards ( $\mathrm{n}=$ 34), Refused access to health care services $(\mathrm{n}=6)$ and Physical Maltreatment $(n=5)$. Combined, this is roughly less than $0.1 \%$ of the respondents. $5.2 \%$ of the respondents said that their personal experience of stigma and discrimination is still continuing.

The Preliminary SADS results for Indonesia reflect a very low level of SAD as experienced by MSM and TG community members. Although packets of SADs, are still being reported, the data suggest an increasing awareness of health care service providers on the concern of SAD and perhaps the effectiveness of government and local NGO-led interventions to significantly decrease if not totally eradicate stigma and discrimination in its many forms.

Disclosure of interest The study was supported by the Global Fund through a regional Grant to the ISEAN Hivos Program. 
The main author has no other affiliations aside from the ISEAN Hivos Program.

\section{O08.5 OUR VOICES, OUR COMMUNITIES, OUR RIGHTS}

Ken Moala*. Technical Advisor, Consultant

\subsection{6/sextrans-2015-052270.125}

Background It has been the desire of the Pacific Sexual Diversity Network (PSDN) since its inception that its network celebrate its identities and ideals in the form of a Human Rights Conference, designed to enhance understanding and transfer knowledge related to LGBTIQ Human Rights. In the Pacific, eight countries criminalise consensual same sex behaviour with many others having related discriminatory laws, and/or laws used with discriminatory and arbitrary application. Even where these laws do not exist, many states have other discriminatory laws that target people because of sexual orientation and gender identity.

Methods Connect people and LGBTIQ organised groups across the Pacific region to share ideas, to affirm the dignity, equality and security of LGBTIQ communities and individuals. Educate and advocate about international human rights law, recent international developments and agreed principles, to enhance respect for persons of diverse sexual orientations and gender identities. Promote collaboration to build genuine and accountable partnerships and networks for advocacy and social action. Promote and enable access to sharing and dissemination of information, ideas, experiences and resources. Improve understanding and strengthen collaborations amongst key stakeholders about health and human rights issues.

Results In assisting local PIDSOGIE communities strengthen knowledge and skills in law and policy reform advocacy, networks such as PSDN have a role in major policy shifts such as the repeal of the Samoan Female Impersonation Law. The 2012 report of the Global Commission on HIV and the Law recognises that good laws fully resourced and rigorously enforced, can protect human rights and widen access to HIV prevention and health services.

Conclusion Community networks such as PSDN make a critical contribution to the development of appropriate and rights based policy and laws at the country level which have a positive impact on the accessibility of prevention and other services for PIDSOGIE communities.

Disclosure of interest statement Pacific Sexual Divesity Network is funded by HIVOS The Netherlands, and Wellsprings, Arcus, Arc International - USA. Pacific Sexual Divesity Network PSDN. Lesbian, Gay, Bisexual, Transgender, Intersex, Queer. LGBTIQ. PIDSOGIE - Pacific Island Diverse Sexual Orientation Gender Indentities and Expressions.

\subsection{THE GENDERED INFLUENCE OF STIGMA ON HIV TESTING BEHAVIOUR: RESULTS FROM A POPULATION- BASED SURVEY OF WOMEN AND MEN IN RWANDA}

${ }^{1} \mathrm{~A}$ Stangl*, ${ }^{1} \mathrm{E}$ Efronson, ${ }^{1} \mathrm{~K}$ Siewwright, ${ }^{2} \mathrm{~S}$ Krishnaratne, ${ }^{2} \mathrm{~J}$ Hargreaves. ${ }^{1}$ The International Center for Research on Women; ${ }^{2}$ The London School of Hygiene and Tropical Medicine

\subsection{6/sextrans-2015-052270.126}

Objective Stigmatisation is a multifaceted process, and distinct domains of stigma may impact HIV testing behaviour differently. We examined the relationship between two stigma domains and
HIV testing behaviour among Rwandan men and women who participated in a population-based survey in 2011.

Methods We conducted multivariable logistic regression with data from 4,669 Rwandan women $(\mathrm{N}=2613)$ and men $(\mathrm{N}=$ 2,056) aged 15 years and older to predict 'ever tested for HIV'. Independent variables included sociodemographics, knowledge of and proximity to HIV, and two stigma domains, 'drivers' (fear of HIV infection through casual contact with PLHIV) and 'manifestations' (anticipated and perceived stigma, shame and discriminatory attitudes). All analyses were disaggregated by gender.

Results Three quarters of women and men reported ever testing for HIV. Sociodemographic variables significantly associated with HIV testing behaviour included: age, secondary (women only) and post-secondary education (both genders), complete knowledge of HIV [women only, OR: 1.52, 95\% CI: 1.20-1.96], frequent trips outside the community (men only), and proximity (personally knowing a PLHIV) [women, OR: 1.66, 95\% CI: 1.22-2.25; men, OR: 1.89 , 95\% CI: 1.36 - 2.60]. Fear of becoming infected with HIV via contact with saliva was the only stigma variable significantly associated with testing behaviour for women [OR: $0.68,95 \%$ CI: 0.49 - 0.94]. For men, holding a discriminatory attitude was the only stigma variable significantly associated with testing behaviour [OR: 0.63, 95\% CI: $0.41-$ 0.98]. Socioeconomic status and residence were not significantly associated with HIV testing behaviour for either gender.

Conclusion These findings demonstrate that drivers and manifestations of stigma influence HIV testing behaviour differently for women and men, suggesting the need for tailored interventions, including stigma-reduction components, to increase HIV testing among both genders in Rwanda. Targeted interventions are also needed to increase testing among adolescents (15-24) and older $(50+)$ men and women.

Disclosure of interest statement Nothing to declare.

\section{9 - Novel methods for STI basic research}

\subsection{STANDARDISED, QUALITY ASSURED TIME-KILL CURVE ANALYSIS AND PHARMACODYNAMIC FUNCTIONS OF DIFFERENT ANTIBIOTICS FOR IN VITRO EVALUATION OF TREATMENT REGIMENS FOR NEISSERIA GONORRHOEAE}

${ }^{1,2,3}$ SF Foerster* ${ }^{3} \mathrm{M}$ Unemo, ${ }^{1} \mathrm{~L}$ Hathaway, ${ }^{2} \mathrm{~N}$ Low, ${ }^{2} \mathrm{CL}$ Althaus. ${ }^{1}$ Institute for Infectious Diseases, University of Bern, Switzerland; 'Institute of Social and Preventive Medicine (ISPM), University of Bern, Switzerland; ${ }^{3}$ WHO Collaborating Centre for Gonorrhoea and Other STIS, National Reference Laboratory for Pathogenic Neisseria, Örebro University Hospital, Örebro, Sweden

\subsection{6/sextrans-2015-052270.127}

Introduction Neisseria gonorrhoeae shows increasing resistance to first line empirical treatment, which demonstrates the need for robust methods to evaluate antibiotic treatment regimens. Antibiotic efficacy is traditionally determined in vitro by measuring minimum inhibitory concentrations (MICs). Time-kill curve assays for N. gonorrhoeae have been difficult to standardise. We developed a new time-kill curve assay and used pharmacodynamics functions to analyse the relationship between antibiotic concentration and bacterial net growth rate.

Methods We used a defined medium (Graver-Wade medium) and grew bacteria in 96-well microtiter plates. To measure colony forming units over a time course of six hours, we used a previously described drop plate method and spotted $10 \mu \mathrm{l}$ droplets on chocolate agar with a multichannel pipette. The assay was 